\title{
Safety and Immunogenicity of Live Oral Cholera Vaccine CVD 103-HgR in Children Aged 2-5 Years in the United States
}

\author{
James M. McCarty, ${ }^{1 \star}$ David Cassie, ${ }^{2}$ Lisa Bedell, ${ }^{2}$ Michael D. Lock, ${ }^{2}$ and Sean Bennett ${ }^{2}$ \\ ${ }^{1}$ Stanford University School of Medicine, Stanford, California; ${ }^{2}$ Emergent Travel Health, Inc., Redwood City, California
}

\begin{abstract}
In a phase 4, randomized, placebo-controlled, double-blind, multicenter study, to assess the safety and immunogenicity of live, attenuated cholera vaccine PXVX0200 in children aged 2-5 years in the United States, 172 volunteers were randomized 6:1 to receive a single dose of $1 \times 10^{9}$ colony forming units (CFU) of PXVX0200 or placebo. Immunogenicity endpoints included serum vibriocidal antibody (SVA) levels on days 1, 11, and 29. Safety was assessed by comparing solicited signs and symptoms on days 1-8, unsolicited adverse events through day 29 , and serious adverse events (SAEs) through day 181. The SVA seroconversion rates 10 days after immunization were $98.1 \%$ and $0 \%$ in vaccine and placebo recipients, respectively, and the vaccine seroconversion rate was non-inferior to the $93.5 \%$ rate seen in the bridging population of adults aged 18-45 years from a lot consistency study. Most reactogenicity was mild to moderate, and there were no study-related SAEs. PXVX0200 appears safe and immunogenic in children aged 2-5 years.
\end{abstract}

\begin{abstract}
Vibrio cholerae remains a major cause of diarrhea in much of the world and in its most severe form (cholera gravis) can lead to severe dehydration and death, an outcome seen most commonly in children younger than 5 years. ${ }^{1}$ It is estimated that 1.3-4 million cases of cholera occur each year and result in up to $21,000-143,000$ deaths worldwide. ${ }^{2}$ Cholera represents an ongoing risk to travelers to countries with both endemic and epidemic disease.
\end{abstract}

PXVX0200 is the research name for Center for Vaccine Development (CVD) 103-HgR, a live attenuated strain of $V$. cholerae O1, for the prevention of cholera diarrhea. It was licensed in the United States in 2016 under the trade name Vaxchora ${ }^{\circledR}$ (Emergent Travel Health Inc., Redwood City, CA) for use in individuals aged 18-64 years traveling to cholera-affected areas. ${ }^{3}$ The safety, efficacy, and immunogenicity of PXVX0200 have been established in adults in four randomized, double-blind, placebocontrolled, multicenter trials. ${ }^{4-7}$ These included a phase 3 cholera challenge study in which PXVX0200 demonstrated $90 \%$ efficacy at 10 days and $80 \%$ efficacy at 3 months versus placebo following ingestion of $1 \times 10^{5} \mathrm{CFU}$ of wild-type $V$. cholerae O1 El Tor Inaba strain N16961. ${ }^{5}$ Because previous studies in children in developing countries of CVD 103-HgR suggested that the immune response, as measured by serum vibriocidal antibody (SVA) seroconversion rates and geometric mean titers (GMTs), was lower than the response in adults, a pediatric trial of PXVX0200 in children was recently performed in the United States. $^{8-10}$ In this study, CVD 103-HgR was shown to be safe, immunogenic, and well tolerated in children and adolescents aged 6-17 years. ${ }^{10}$ Here, we report for the first time the safety and immunogenicity results from cohort 3 of that study, that is, children aged $2-5$ years.

This was a multicenter phase 4 randomized, double-blind, placebo-controlled trial designed to assess the immunogenicity, safety, palatability, and acceptability of PXVX0200 in children aged 2-17 years. Study methods have been previously described and were similar for cohort 3, except that palatability was assessed by the caregiver instead of the subject. ${ }^{10}$ The study was performed in children aged $2-5$ years

*Address correspondence to James M. McCarty, Stanford University School of Medicine, 291 Campus Dr., Stanford, CA 94305. E-mail: jmccart2@stanford.edu at eight U.S. sites from July 2017 through September 2019. For serum antibody assays, blood was collected from all participants on days 1, 11, and 29. For cohort 3, vaccine was prepared as previously described, except that $50 \mathrm{~mL}$ of buffer solution was used with each dose, rather than $100 \mathrm{~mL}$. Placebo consisted of $50 \mathrm{~mL}$ of physiological (0.9\%) saline. The primary endpoint was the proportion of participants achieving seroconversion, defined as a 4-fold or greater rise over baseline SVA, at day 11 after one dose of PVX0200 in the immunogenicity evaluable population (IEP), which included the set of participants who received vaccination, consumed at least $80 \%$ of the study treatment, had evaluable results from both day 1 and day 11, and had no major protocol violations that would affect immunogenicity. The co-primary immunogenicity objectives were to demonstrate a minimum seroconversion rate of $70 \%$ and non-inferiority to the seroconversion rate in the bridging population of adults aged 18-45 years from the lot consistency study. ${ }^{6}$

A total of 187 participants aged 2-5 years were screened, 176 were randomized across the sites, and 155 completed the study through the day 181 visit (Figure 1). The demographic and baseline characteristics of the two groups were similar and, other than age, were also similar to cohorts 1 and 2 . The day 11 SVA seroconversion rate in the IEP of cohort 3 was $98.1 \%$, which was non-inferior to the $93.5 \%$ rate in the adult bridging population and was also greater than $70 \%$ (Table 1). Thus, both primary immunogenicity objectives were met. No additional seroconversions occurred between day 11 and day 29. The SVA GMTs of cohort 3 peaked at 4,852 on day 11 and decreased to 1,014 by day 29 , whereas the geometric mean fold increase (GMFI) of serum vibriocidal antibodies peaked at 182 on day 11 and decreased to 38 by day 29 . The incidence of vomiting was higher in the placebo group; otherwise there were no significant differences in the frequency and severity of solicited reactogenicity signs and symptoms between PXVX0200 and placebo recipients (Table 2). There was one serious adverse event (SAE), a hospitalization for an asthma exacerbation and pneumonia, in a placebo subject, and one "life-threatening" fever $\left(>40^{\circ} \mathrm{C}\right.$, per protocol definition) in a PXVX0200 subject, which were both considered not related to study product.

Of the 172 cohort 3 subjects dosed, $\geq 80 \%$ of the dose was consumed by $82.7 \%$ and $84.6 \%$ of Vaxchora and placebo 

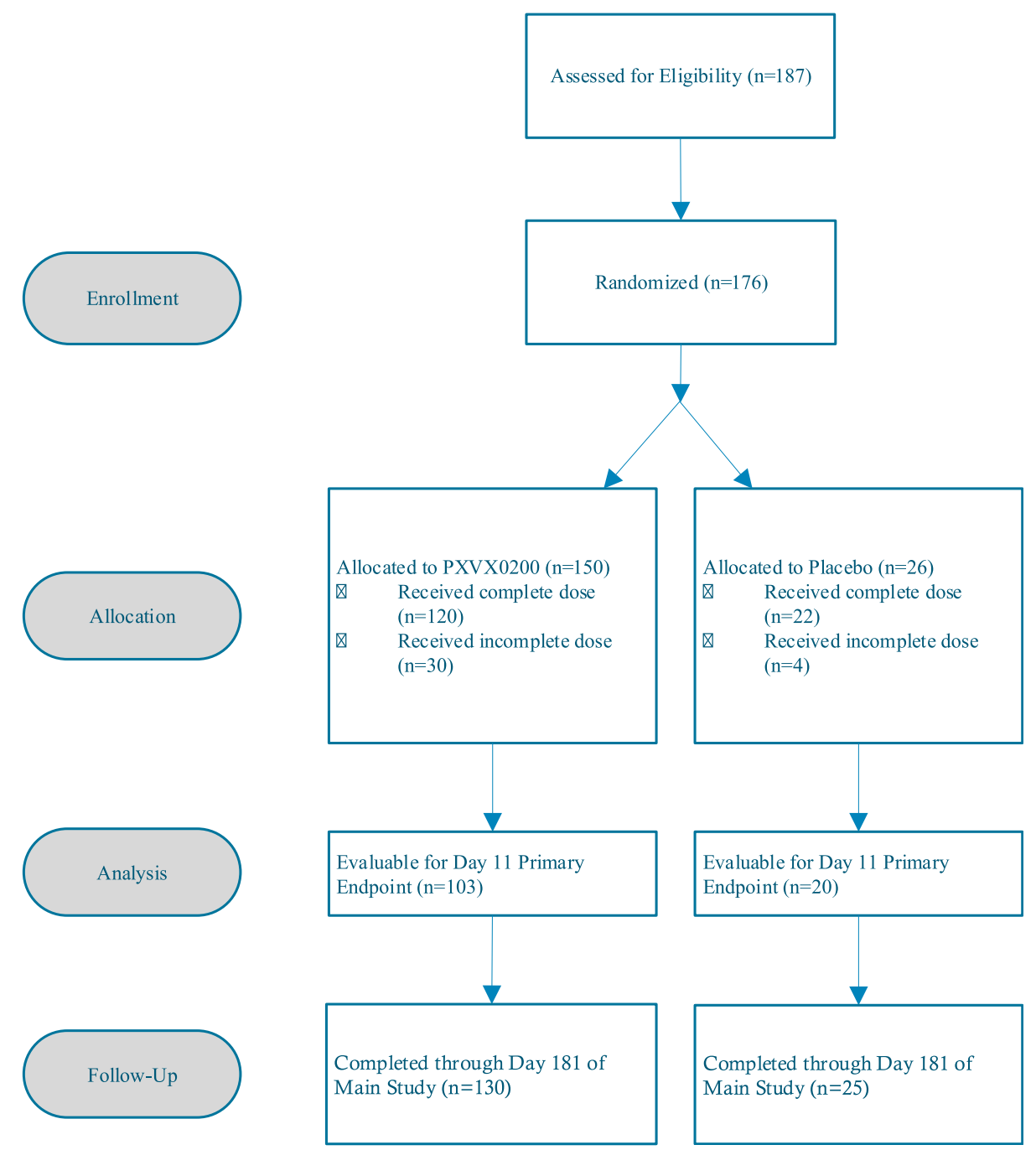

FIGURE 1. Participant disposition. Consolidated Standards of Reporting Tools diagram for pediatric trial: Reasons for withdrawal were as follows: four participants withdrew consent, 14 were lost to follow-up, and three were noncompliant with the protocol. This figure appears in color at www.ajtmh.org.

recipients, respectively. This was less than the $99.4 \%$ and $93.7 \%$ of vaccine recipients in the 12 - to 17 - and 6 - to 11 -yearold cohorts, respectively, who consumed $\geq 80 \%$ of the dose. ${ }^{10}$ Taste was ranked as very good, good, or neutral by the caregivers of $91(62.3 \%)$ and $18(69.2 \%)$ of Vaxchora and placebo recipients, respectively. Optional Pure Via Stevia sweetener was added to all, but one, of the doses; thus, assessment of its effect on palatability was not possible.

Cholera is a threat to children and adults who travel to endemic areas and can cause severe and fatal disease. A single dose vaccine that can provide rapid and effective immunity would be ideal. Here, we document the safety, immunogenicity, and tolerability of single-dose, live oral cholera vaccine PXVX0200 in children aged 2-5 years in a developed country. Serum vibriocidal antibody seroconversion was measured as the primary endpoint in this bridging study comparing rates in children with those in adults aged 18-45 years. ${ }^{6}$ In cohort 3, seroconversion occurred in $98.1 \%$ of vaccine recipients aged $2-5$ years versus $0 \%$ in placebo recipients $(P<0.0001)$. These rates were non-inferior to the $93.5 \%$ seroconversion rate in the adult bridging population of the phase 3 lot consistency study, and because the lower limit of the $98.3 \% \mathrm{Cl}$ for seroconversion was greater than $70 \%$, both primary objectives were met. Because SVA seroconversion was shown to be a strong correlate of protection against moderate to severe cholera diarrhea in the adult challenge study, it is possible that children aged 2-5 years vaccinated with PXVX0200 will be protected against cholera-induced diarrhea. ${ }^{5,11}$ In this study, GMT and GMFI at day 11 were lower than those seen in cohorts 1 and 2 and the adult bridging study, possibly reflecting immunologic or intestinal immaturity in the youngest cohort. ${ }^{6,10}$ However, because seroconversion rates were similar and SVA seroconversion is the best correlate of protection against moderate to severe diarrhea, it is hoped that because challenge studies cannot be performed in children, the efficacy of PXVX0200 in the youngest cohort would be similar to the efficacy in adults.

Previous studies of single-dose CVD 103-HgR oral cholera vaccine in developing countries demonstrated that SVA seroconversion rates were lower in children than those seen in adults, and higher dose formulations with $5 \times 10^{9} \mathrm{CFU}$ were developed, with seroconversion seen in only $22-78 \%$ of 
TABLE 1

Seroconversion, GMT, and fold increase for children and adults

\begin{tabular}{|c|c|c|c|c|c|c|c|c|}
\hline & \multicolumn{2}{|c|}{ Adults (18-45 years) } & \multicolumn{2}{|c|}{ Cohort 1 (12-17 years) } & \multicolumn{2}{|c|}{ Cohort 2 (6-11 years) } & \multicolumn{2}{|c|}{ Cohort 3 ( $2-5$ years) } \\
\hline & $\begin{array}{l}\text { PXVX0200 } \\
(n=2,688)\end{array}$ & $\begin{array}{l}\text { Placebo } \\
(N=334)\end{array}$ & $\begin{array}{l}\mathrm{PXVX0200} \\
(n=157)\end{array}$ & $\begin{array}{l}\text { Placebo } \\
(n=23)\end{array}$ & $\begin{array}{l}\mathrm{PXVX0200} \\
(n=139)\end{array}$ & $\begin{array}{l}\text { Placebo } \\
(n=24)\end{array}$ & $\begin{array}{l}\mathrm{PXVX0200} \\
(n=103)\end{array}$ & $\begin{array}{l}\text { Placebo } \\
(n=20)\end{array}$ \\
\hline \multicolumn{9}{|l|}{ Seroconversion } \\
\hline \multicolumn{9}{|l|}{ Day 11 visit } \\
\hline$N$ analyzable & 2,687 & - & 157 & - & 139 & - & 103 & - \\
\hline$N(\%)$ seroconverted & $2,513(93.5)$ & - & $156(99.4 \%)$ & - & $136(97.8 \%)$ & - & $101(98.1)$ & - \\
\hline $98.3 \% \mathrm{Cl}$ & {$[92.3 \%, 94.6 \%]$} & - & {$[95.4 \%, 99.9 \%]$} & & {$[92.5 \%, 99.4 \%]$} & - & {$[91.5 \%, 99.6 \%]$} & - \\
\hline $\begin{array}{l}\text { Difference (cohort minus } \\
\text { adults) }\end{array}$ & - & - & $5.8 \%$ & - & $4.3 \%$ & - & $4.5 \%$ & - \\
\hline $96.7 \% \mathrm{Cl}$ on difference & - & - & {$[2.4 \%, 7.1 \%]$} & - & {$[-0.3 \%, 6.2 \%]$} & - & {$[-1.1 \%, 6.4 \%]$} & - \\
\hline$P$-value & - & - & 0.0009 & - & 0.0455 & - & 0.0628 & - \\
\hline \multicolumn{9}{|l|}{ GMT } \\
\hline \multicolumn{9}{|l|}{ Day 1 visit } \\
\hline$N$ analyzable & 2,687 & 334 & 157 & 23 & 139 & 24 & 103 & 20 \\
\hline GMT & 69 & 72 & 32 & 44 & 32 & 36 & 27 & 26 \\
\hline $95 \% \mathrm{Cl}$ & {$[65,72]$} & {$[62,84]$} & {$[28,37]$} & {$[28,70]$} & {$[28,36]$} & {$[24,52]$} & {$[24,30]$} & {$[19,36]$} \\
\hline Minimum, maximum & $20,10,240$ & $20,20,480$ & 20,640 & $20,1,280$ & $20,1,280$ & 20,640 & 20,320 & 20,320 \\
\hline \multicolumn{9}{|l|}{ Day 11 visit } \\
\hline$N$ analyzable & 2,688 & 334 & 157 & 23 & 139 & 24 & 103 & 20 \\
\hline GMT & 9,688 & 85 & $8,735^{\star}$ & 41 & $8,305^{\star}$ & 40 & $4,852^{*}$ & 28 \\
\hline $95 \% \mathrm{Cl}$ & {$[9,067,10,351]$} & {$[72,101]$} & {$[7,053,10,819]$} & {$[26,65]$} & {$[6,516,10,586]$} & {$[23,70]$} & {$[3,445,6,832]$} & {$[20,39]$} \\
\hline Minimum, maximum & $20,327,680$ & $20,40,960$ & $20,81,920$ & $20,1,280$ & $40,163,840$ & $20,5,120$ & $20,163,840$ & 20,320 \\
\hline \multicolumn{9}{|l|}{ Day 29 visit } \\
\hline$N$ analyzable & $26 \dagger$ & $6 \dagger$ & 156 & 23 & 138 & 23 & 98 & 18 \\
\hline GMT & 6,170 & 127 & $2,749^{\star}$ & 43 & $1,952^{*}$ & 40 & $1,014^{*}$ & 27 \\
\hline $95 \% \mathrm{Cl}$ & {$[3,798,10,025]$} & {$[10,1,623]$} & {$[2,311,3,270]$} & {$[27,67]$} & {$[1,554,2,452]$} & {$[22,72]$} & {$[741,1,387]$} & {$[21,36]$} \\
\hline Minimum, maximum & $640,40,960$ & $20,5,120$ & $320,20,480$ & 20,640 & $40,40,960$ & $20,5,120$ & $20,40,960$ & 20,80 \\
\hline \multicolumn{9}{|c|}{ Geometric mean fold increase } \\
\hline \multicolumn{9}{|l|}{ Day 11 visit } \\
\hline$N$ analyzable & - & - & 157 & 23 & 139 & 24 & 103 & 20 \\
\hline Mean fold increase & - & - & $272^{*}$ & 1 & $264^{*}$ & 1 & $182^{\star}$ & 1 \\
\hline $95 \% \mathrm{Cl}$ & - & - & {$[222,334]$} & {$[1,1]$} & {$[204,341]$} & {$[1,2]$} & {$[131,252]$} & {$[1,1]$} \\
\hline Minimum, maximum & - & - & $1,4,096$ & 0,2 & $1,4,096$ & 1,256 & $1,4,096$ & 1,2 \\
\hline \multicolumn{9}{|l|}{ Day 29 visit } \\
\hline$N$ analyzable & - & - & 156 & 23 & 138 & 23 & 98 & 18 \\
\hline Mean fold increase & - & - & $85^{\star}$ & 1 & $62^{*}$ & 1 & $38^{\star}$ & 1 \\
\hline $95 \% \mathrm{Cl}$ & - & - & {$[72,102]$} & {$[1,1]$} & {$[49,78]$} & {$[1,2]$} & {$[28,51]$} & {$[1,1]$} \\
\hline Minimum, maximum & - & - & $8,1,024$ & 0,2 & $1,1,024$ & 1,32 & 1,512 & 0,2 \\
\hline
\end{tabular}

${ }^{\star} P<0.0001 ; P$-values are based on $\mathrm{t}$-statistics assuming normal distribution of the log titer.

† Data for adults for PXVX0200 and placebo from the immune substudy population.

vaccine recipients at this dose. ${ }^{8,9,12-17} \mathrm{~A}$ large efficacy trial of CVD 103-HgR in Indonesia failed to demonstrate protection in children, although herd immunity may have contributed to the unexpected reduction of cholera cases in placebo recipients in that study. ${ }^{2,16}$ A mass vaccination campaign using singledose CVD 103-HgR during a cholera outbreak in Micronesia, which included children aged 2-5 years, demonstrated a vaccine efficacy of $79.2 \%{ }^{21}$ The reduced immune responses in developing countries may be due to multiple factors, including malnutrition, small bowel overgrowth with intestinal mucosal damage (chronic environmental enteropathy), and preexisting immunity due to natural exposure to cholera infection. ${ }^{18,19}$ None of these conditions are common in the United States where children mount a more robust immune response to live oral cholera vaccine.

The study vaccine was well accepted by $82.7 \%$ of cohort 3 subjects versus $99.4 \%$ and $93.7 \%$, respectively, of subjects in cohorts 1 and 2 , and by $84.6 \%$ of placebo recipients in cohort 3 , further demonstrating the impact of age on the willingness and ability to consume the oral solution. ${ }^{10}$ As noted in cohorts 1 and 2, there was no significant difference in palatability assessments between vaccine and placebo recipients in cohort 3. Previously in a trial of CVD 103-HgR in infants and toddlers in Chile, similar seroconversion rates occurred in participants who consumed partial doses of vaccine versus full doses. ${ }^{15}$ However, these results may not be applicable to the current study.

The data from this study demonstrate that PXVX0200 is well tolerated in children aged 2-5 years, with a safety profile similar to subjects aged 6-17 years and adults aged 18-45 years in the lot consistency study. Other than vomiting, there were no significant differences in the incidence of solicited reactogenicity, including diarrhea or fever, between vaccine and placebo recipients. One vaccine recipient developed a fever which was classified as "life-threatening" by predefined criteria but was not considered related to study product. There were no vaccine-related SAEs in any pediatric cohort.

Two other cholera vaccines are available outside of the United States, whole cell recombinant B subunit (Dukoral ${ }^{\circledR}$, SBL Vaccin AB, Stockholm, Sweden) and bivalent, killed whole cell vaccine $\left(\right.$ Shanchol ${ }^{\circledR}$, Shantha Biotechnics Limited, Andhra Pradesh, India, Euvichol ${ }^{\circledR}$, Eubiologics, Seoul, South Korea) administered as two or three doses depending on age. ${ }^{20}$ Because SVA seroconversion has been demonstrated in up to $80 \%$ of adult subjects in as little as 7 days after administration of PXVX0200, single-dose administration of 
TABLE 2

Solicited adverse events

\begin{tabular}{|c|c|c|c|c|c|c|c|c|}
\hline \multirow[b]{2}{*}{ Solicited event [ $n(\%)]$} & \multicolumn{2}{|c|}{ Cohort 1 (12-17 years) } & \multicolumn{2}{|c|}{ Cohort 2 (6-11 years) } & \multicolumn{2}{|c|}{ Cohort 3 ( $2-5$ years) } & \multicolumn{2}{|c|}{ Overall (2-17 years) } \\
\hline & $\begin{array}{c}P X V X 0200 \\
(N=165)\end{array}$ & $\begin{array}{l}\text { Placebo } \\
(N=24)\end{array}$ & $\begin{array}{c}\text { PXVX0200 } \\
(N=157)\end{array}$ & $\begin{array}{l}\text { Placebo } \\
(N=25)\end{array}$ & $\begin{array}{c}\text { PXVX0200 } \\
(n=146)\end{array}$ & $\begin{array}{l}\text { Placebo } \\
(n=26)\end{array}$ & $\begin{array}{c}\mathrm{PXVX0200} \\
(n=468)\end{array}$ & $\begin{array}{l}\text { Placebo } \\
(n=75)\end{array}$ \\
\hline Any event & $113(68.5)$ & $16(66.7)$ & $86(54.8)$ & $13(52.0)$ & $59(40.4)$ & $9(34.6)$ & $258(55.1)$ & $38(50.7)$ \\
\hline Mild/moderate & 109 (66.1) & $15(62.5)$ & $81(51.6)$ & $13(52.0)$ & $58(39.7)$ & $8(30.8)$ & $248(53.0)$ & 36 (48.0) \\
\hline Severe & $4(2.4)^{\star}$ & $1(4.2)$ & $5(3.2)$ & 0 & $1(0.7)^{\star}$ & $1(3.8)$ & $10(2.1) \dagger$ & $2(2.7)$ \\
\hline Tiredness & $67(40.6)$ & $9(37.5)$ & $55(35.0)$ & $8(32.0)$ & $45(30.8)$ & $6(23.1)$ & $167(35.7)$ & $23(30.7)$ \\
\hline Mild/moderate & $66(40.0)$ & 8 (33.3) & $54(34.4)$ & 8 (32.0) & $45(30.8)$ & $6(23.1)$ & 165 (35.3) & $22(29.4)$ \\
\hline Severe & $1(0.6)^{*}$ & $1(4.2)$ & $1(0.6)$ & 0 & 0 & 0 & $2(0.4) \dagger$ & 1 (1.3) \\
\hline Headache & $74(44.8)$ & $11(45.8)$ & $41(26.1)$ & $6(24.0)$ & $13(8.9)$ & $2(7.7)$ & $128(27.4)$ & $19(25.3)$ \\
\hline Mild/moderate & 73 (44.2) & $11(45.8)$ & $39(24.8)$ & $6(24.0)$ & 13 (8.9) & $2(7.6)$ & $125(26.7)$ & 19 (25.4) \\
\hline Severe & $1(0.6)$ & 0 & $2(1.3)$ & 0 & 0 & 0 & $3(0.6)$ & 0 \\
\hline Abdominal pain & $62(37.6)$ & $4(16.7)$ & $43(27.4)$ & $6(24.0)$ & 25 (17.1) & $4(15.4)$ & $130(27.8)$ & $14(18.7)$ \\
\hline Mild/moderate & $61(37.0)$ & $4(16.7)$ & $43(27.4)$ & $6(24.0)$ & $25(17.1)$ & $4(15.4)$ & $129(27.6)$ & $14(18.7)$ \\
\hline Severe & $1(0.6)$ & 0 & 0 & 0 & 0 & 0 & $1(0.2)$ & 0 \\
\hline Lack of appetite & $48(29.1)$ & $3(12.5)$ & 24 (15.3) & $5(20.0)$ & 28 (19.2) & $3(11.5)$ & $100(21.4)$ & $11(14.7)$ \\
\hline Mild/moderate & $48(29.1)$ & $3(12.5)$ & $23(14.6)$ & $5(20.0)$ & 28 (19.1) & $3(11.5)$ & 99 (21.2) & $11(14.7)$ \\
\hline Severe & 0 & 0 & $1(0.6)$ & 0 & 0 & 0 & $1(0.2)$ & 0 \\
\hline Nausea & $37(22.4)$ & $6(25.0)$ & $22(14.0)$ & $4(16.0)$ & $10(6.8)$ & $4(15.4)$ & $69(14.7)$ & $14(18.7)$ \\
\hline Mild/moderate & $36(21.8)$ & $6(25.0)$ & $22(14.0)$ & $4(16.0)$ & $10(6.9)$ & $4(15.4)$ & 68 (14.5) & $14(18.7)$ \\
\hline Severe & $1(0.6)$ & 0 & 0 & 0 & 0 & 0 & $1(0.2)$ & 0 \\
\hline Vomiting & $9(5.5)$ & 0 & $7(4.5)$ & 0 & $2(1.4)$ & $3(11.5)$ & $18(3.8)$ & $3(4.0)$ \\
\hline Mild/moderate & $8(4.8)$ & 0 & $7(4.5)$ & 0 & $2(1.4)$ & $3(11.5)$ & $17(3.6)$ & $3(4.0)$ \\
\hline Severe & $1(0.6)$ & 0 & 0 & 0 & 0 & 0 & $1(0.2)$ & 0 \\
\hline Fever & $2(1.2)$ & 0 & $5(3.2)$ & $1(4.0)$ & $3(2.1)$ & $1(3.8)$ & $10(2.1)$ & $2(2.7)$ \\
\hline Mild/moderate & $1(0.6)$ & 0 & $1(0.6)$ & $1(4.0)$ & $2(1.4)$ & 0 & $4(0.9)$ & 1 (1.3) \\
\hline Severe & $1(0.6)$ & 0 & $4(2.5)$ & 0 & $1(0.7)^{\star}$ & $1(3.8)$ & $6(1.3) \dagger$ & 1 (1.3) \\
\hline Diarrhea & $6(3.6)$ & $1(4.2)$ & 0 & 0 & $1(0.7)$ & 0 & $7(1.5)$ & 1 (1.3) \\
\hline Mild/moderate & 3 (1.8) & 1 (4.2) & 0 & 0 & $1(0.7)$ & 0 & $4(0.9)$ & 1 (1.3) \\
\hline Severe & $3(1.8)$ & 0 & 0 & 0 & 0 & 0 & $3(0.6)$ & 0 \\
\hline
\end{tabular}

(cohort 1) not related to study product.

† Includes one case each of potentially life-threatening fever or tiredness.

PXVX0200 could offer a potential advantage in an outbreak situation in a vulnerable pediatric population. ${ }^{5}$ An additional study beginning in 2021 will assess the safety and immunogenicity of PXVX0200 in infants aged six through 23 months.

PXVX0200 is safe, immunogenic, and well tolerated in children aged 2-5 years in developed countries and may provide rapid protection in a single dose against cholera diarrhea in those traveling to or residing in at-risk countries.

Received July 28, 2020. Accepted for publication November 7, 2020. Published online December 14, 2020

Authors' addresses: James M. McCarty, Department of Pediatrics, Stanford University School of Medicine, Stanford, CA, E-mail: jmccart2@stanford.edu. David Cassie, Clinical Research, Emergent BioSolutions Canada, Inc., Winnipeg, Canada, E-mail: dcassie@ ebsi.com. Lisa Bedell and Michael D. Lock, Department of Biostatistics, Emergent Travel Health, Inc., Redwood City, CA, E-mails: bedelll@ebsi.com and mike@lockstats.com. Sean Bennett, Clinical Development, Emergent Travel Health, Inc., Redwood City, CA, E-mail: sbennett22903@gmail.com.

This is an open-access article distributed under the terms of the Creative Commons Attribution (CC-BY) License, which permits unrestricted use, distribution, and reproduction in any medium, provided the original author and source are credited.

\section{REFERENCES}

1. Weil AA, Ryan ET, 2018. Cholera: recent updates. Curr Opin Infect Dis 31: 455-466.

2. Ali M, Nelson AR, Lopez AL, Sack DA, 2015. Updated global burden of cholera in endemic countries. PLoS Negl Trop Dis 9: e0003832.
3. Wong KK, Burdette E, Mahon BD, Mintz ED, Ryan ET, Reingold AL, 2017. Recommendations of the advisory committee on immunization practices for use of cholera vaccine. MMWR Morb Mortal Wkly Rep 66: 482-485.

4. Chen WH, Greenberg RN, Pasetti MF, Livio S, Lock M, Gurwith M, Levine MM, 2014. Safety and immunogenicity of single-dose live oral cholera vaccine strain CVD 103-HgR, prepared from new master and working cell banks. Clin Vaccine Immunol 21: 66-73.

5. Chen WH et al., 2016. Single-dose live oral cholera vaccine CVD 103-HgR protects against human experimental infection with Vibrio cholerae O1 EI Tor. Clin Infect Dis 62: 1329-1335.

6. McCarty JM, Lock MD, Hunt KM, Simon JK, Gurwith M, 2018. Safety and immunogenicity of single-dose live oral cholera accine strain CVD 103-HgR in healthy adults age 18-45. Vaccine 36: 833-840.

7. McCarty JM, Lock MD, Bennett S, Hunt KM, Simon JK, Gurwith $M, 2019$. Age related immunogenicity and reactogenicity of live oral cholera vaccine CVD 103-HgR in a randomized, controlled clinical trial. Vaccine 37: 1389-1397.

8. Suharyono et al., 1992. Safety and immunogenicity of single-dose live oral cholera vaccine CVD 103-HgR in 5-9-year-old Indonesian children. Lancet 340: 689-694.

9. Simanjuntak $\mathrm{CH}$ et al., 1993. Safety, immunogenicity, and transmissibility of single-dose live oral cholera vaccine strain CVD 103-HgR in 24- to 59-month-old Indonesian children. J Infect Dis 168: 1160-1176.

10. McCarty JM, Gierman EC, Bedell L, Lock MD, Bennet S, 2020. Safety and immunogenicity of live oral cholera vaccine CVD 103-HgR in children and adolescents aged 6-17 Years. Am J Trop Med Hyg 102: 48-57.

11. Haney DJ, Lock MD, Simon JK, Harris J, Gurwith M, 2017. Antibody-based correlates of protection against cholera: analysis of a challenge study in a cholera-naïve population. Clin Vaccine Immunol 24: e00098-e00117.

12. Lagos R, Avendano A, Prado V, Horwitz I, Wasserman S, Losonsky G, 1995. Attenuated live cholera vaccine strain CVD 
103-HgR elicits significantly higher serum vibriocidal antibody titers in persons of blood group O. Infect Immun 62: 702-709.

13. Lagos R, Losonsky G, Abrego P, San Martin O, Prado V, Wasserman S, Levine MM, 1996. Tolerance, immunogenicity, excretion and transmission of the live-attenuated cholera vaccine CVD 103-HgR. Double blind paired study in Chilean 24-to 59-month old children. Bol Med Hosp Infant Mex 53: 214-220.

14. Lagos R, Fasano A, Wasserman SS, Prado V, San Martin O, Abrego P, Losonsky GA, Alegria S, Levine MM, 1999. Effect of small bowel bacterial overgrowth on the immunogenicity of single-dose live oral cholera vaccine CVD 103-HgR. J Infect Dis 180: 1709-1712.

15. Lagos R, Martin OS, Wasserman SS, Prado V, Losonsky GA, Bustamante C, Levine MM, 1999. Palatability, reactogenicity and immunogenicity of engineered live oral cholera vaccine CVD 103-HgR in Chilean infants and toddlers. Pediatr Infect Dis J 18: 624-630.

16. Richie E, Punjabi NH, Sidharta Y, Peetosutan K, Sukandar M, Wasserman SS, 2000. Efficacy trial of single -dose live oral cholera vaccine CVD 103-HgR in North Jakarta, Indonesia, a cholera endemic area. Vaccine 18: 2399-2410.

17. Faucher JF, Binder R, Missinou MA, Matsiegui PB, Grus $H$, Neubauer R, 2002. Efficacy of atovaquone/proguanil for malaria prophylaxis in children and its effect on the immunogenicity of life oral typhoid and cholera vaccines. Clin Infect Dis 35: 1147-1154.

18. Sack DA, Qadri F, Svennerholm AM, 2008. Determinants of responses to oral vaccines in developing countries. Ann Nestle [Engl] 66: 71-79.

19. Levine MM, 2010. Immunogenicity and efficacy of oral vaccines in developing countries: lessons from a live cholera vaccine. $B M C$ Biol 8: 129.

20. Saha A, Rosewell A, Hayen A, Maclntyre CR, Qadri F, 2017. Improving immunization approaches to cholera. Expert Rev Vaccines 16: 235-248.

21. Calain $P$, Chaine JP, Johnson E, Hawley ML, O'Leary MJ, Oshitani $\mathrm{H}$, Chaignat $\mathrm{CL}, 2004$. Can oral cholera vaccination play a role in controlling a cholera outbreak? Vaccine 22: 2444-2451. 